\title{
Cosmic-ray variations in the solar neighbourhood
}

\author{
Isabelle A. Grenier, ${ }^{a, *}$ François R. Kamal Youssef ${ }^{a}$ and M. Nicola Mazziotta ${ }^{b}$ \\ ${ }^{a}$ Université de Paris and Université Paris Saclay, CEA, CNRS, AIM, \\ CEA Saclay, F-91190 Gif-sur-Yvette, France \\ ${ }^{b}$ Istituto Nazionale di Fisica Nucleare, Sezione di Bari, \\ via Orabona 4, I-70125 Bari, Italy \\ E-mail: isabelle.grenier@cea.fr, francois.kamalyoussef@cea.fr, \\ Marionicola.Mazziotta@ba.infn.it
}

The $\gamma$ radiation produced in cosmic-ray and gas interactions has been used to probe the local cosmic-ray spectrum in several clouds to distances of $\sim 1 \mathrm{kpc}$ around the Sun. Early measurements made with Fermi LAT data showed consistent gas emissivities across this region and good agreement with the cosmic-ray spectra reaching the Solar System, given the 10-15\% uncertainty in gauging the atomic gas mass pervaded by the cosmic rays. The local cosmic-ray uniformity has recently been challenged by the finding of a significant loss of particles in the nearby Eridu cirrus with respect to the 1-kpc-wide average. We now aim to compare the CR flux in Eridu with that near the Sun and in clouds at comparably short distances. We have therefore revisited the $\gamma$-ray emissivity spectrum one would expect per gas nucleon from hadronic and bremsstrahlung emission using the latest cosmic-ray spectra inferred near the Sun, outside the heliosphere. We have also used improved photon production spectra in nucleus-nucleus interactions. We find that, because of the current uncertainty in the $\gamma$-ray yield per nuclear interaction, one cannot conclude whether the average cosmic-ray spectrum measured within $\sim 1 \mathrm{kpc}$ from the Sun is comparable or superior to that inferred just outside the Solar System, nor whether the low cosmic-ray flux pervading the Eridu cirrus is consistent or not with the flux reaching the Sun. The Eridu cirrus and the Sun both lie in a hollow valley of the local interstellar medium. We show that the cosmic-ray flux in Eridu is about half that observed in two other more massive clouds flanking the valley, 200-300 pc away from Eridu.

$37^{\text {th }}$ International Cosmic Ray Conference (ICRC 2021)

July 12th - 23rd, 2021

Online - Berlin, Germany

\footnotetext{
*Presenter
} 
Most of the Galactic interstellar $\gamma$ rays observed by Fermi LAT in the $10^{8}-10^{11} \mathrm{eV}$ band originate from nuclear interactions between cosmic rays (CR) and interstellar gas. Below $300 \mathrm{MeV}$, electron bremsstrahlung radiation also contributes to the gas emission, but is difficult to separate because of source confusion given the LAT angular resolution at low energy. Above $300 \mathrm{MeV}$, the $\gamma$-ray emissivity spectra obtained per gas nucleon in different nearby clouds show a rather uniform distribution. The sample shows less than $30 \%$ variations in amplitude and the same spectrum across the $\gamma$-ray band (see Figure 4a of [1]). Those measurements were made in clouds within a few hundred parsecs from the Sun and at low altitude in the disc $(|z| \lesssim 100 \mathrm{pc})$. They were made in the atomic phase of the gas where the mass threaded by CR can be directly estimated, unlike in the molecular phase. Yet residual systematic uncertainties due to self absorption of the Hi lines in the densest atomic parts can limit the interpretation of amplitude variations in $\gamma$-ray emissivity. Claims of significant CR variations in flux or spectrum in the local interstellar medium (ISM), [e.g. 2, 3], have severely overlooked interstellar complications (cloud confusion along sightlines, non uniform conversions of $\mathrm{CO}$ line intensities to $\mathrm{H}_{2}$ column densities, masses of optically-thick $\mathrm{HI}$ and $\mathrm{CO}$-dark $\mathrm{H}_{2}$, severe non-linearities in the use of dust optical depths to trace the total gas, etc). The CR homogeneity near the Sun was, however, significanty challenged by the study of the light Eridu Hi cirrus, at a height of 200-250 pc below the Galactic plane, which presents a $34 \%$ lower $\gamma$-ray emissivity, but similar spectrum, than the local ISM average [4]. This emissivity is at variance with the dispersion seen among other nearby clouds and with the CR flux decline expected towards the halo (see Figure 13 of [4]). The emissivity of the Eridu cirrus was compared to the average [15] found in the atomic gas at latitudes $10^{\circ} \leq|b| \leq 70^{\circ}$ which span distances to typically $1 \mathrm{kpc}$. Here we compare the Eridu emissivity to more local measurements, i.e. to the local interstellar spectra (LIS) of CR incident on the heliosphere, and to emissivities in specific clouds that outline the contrasted structure of the local ISM.

\section{Hadronic and bremsstrahlung cross sections}

The $\gamma$-ray yields produced by inelastic interactions between different $\mathrm{CR}$ and gas nuclei have been calculated with the FLUKA simulation package [5] and, alternatively, with a combination of the QGSJET-II-04m model [6] and of the "Kamae" parametrisation for p-H interactions [7]. We refer to these calculations as 'FLUKA' and 'K+QGSJET', respectively. The FLUKA simulation has been benchmarked with a large set of experimental data collected in accelerator experiments and it notably includes prompt pion production as well as secondary production from short-lived particles in the nuclear interaction. We have produced cross sections for the main $\mathrm{p}-\mathrm{H}, \mathrm{p}-\mathrm{He}, \mathrm{He}-\mathrm{H}$, and He-He interactions. The results of the QGSJET-II-04m model have been tuned to LHCf, LHCb, and NA61 data at kinetic energies of tens to hundreds of GeV. Following [8], we have restricted the use of the QGSJET matrices provided in [6] to kinetic energies $E_{k}>30 \mathrm{GeV}$ per nucleon and we have scaled the Kamae matrix to the QGSJET ones in the $30 \leq E_{k} \leq 100 \mathrm{GeV} / \mathrm{n}$ range. To extend the QGSJET matrices at lower energies for CRs heavier than protons, we have exploited the fact that these cross-sections, when expressed per nucleon-nucleon interaction and in kinetic energy per nucleon, closely follow the $\mathrm{p}-\mathrm{H}$ one in shape and values. The resulting nuclear enhancement factors vary with both the nucleon and photon energies. They agree to better than $10 \%$ with the Mori DMPJET-3 estimates [9] in the $10-1000 \mathrm{GeV} / \mathrm{n}$ range for $1 \mathrm{GeV}$ photons. The agreement degrades 


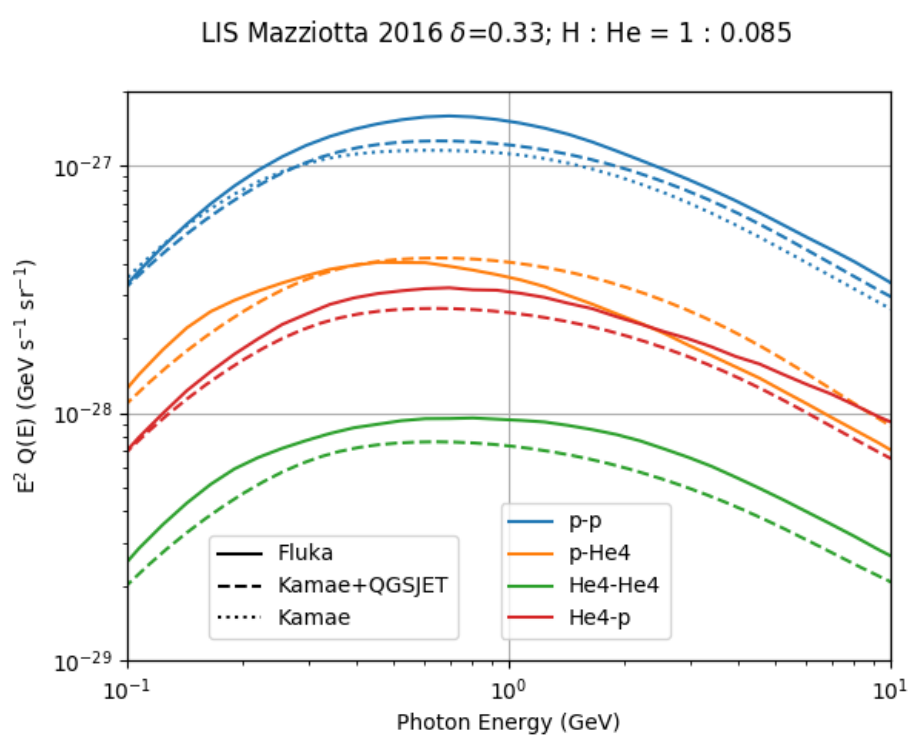

Figure 1: Comparison of the $\gamma$-ray yields obtained for the main p-H, p-He, He-H, and He-He interactions with the FLUKA and K+QGSJET cross-sections assuming the same input CR spectra from [5].

at higher photon energies. [6] provides cross sections for the main p-H, p-He, $\mathrm{He}-\mathrm{H}, \mathrm{He}-\mathrm{He}, \mathrm{C}-\mathrm{H}$, $\mathrm{Al}-\mathrm{H}$, and Fe-H interactions. Since we have solar-demodulated CR spectra for all nuclei with charge number $Z \leq 28$, we have interpolated this set of cross sections (per nucleon-nucleon interaction and in kinetic energy per nucleon) to produce matrices for the missing interactions. The FLUKA calculations were run .

Figure 1 compares the $\gamma$-ray yield obtained for the four main $\mathrm{p}-\mathrm{H}, \mathrm{p}-\mathrm{He}, \mathrm{He}-\mathrm{H}$, and $\mathrm{He}-\mathrm{He}$ interactions with the FLUKA and K+QGSJET sets of cross-sections, assuming the same input CR spectra from [5]. The maximum deviations reach 26\%, 38\%, 21\%, and 34\%, respectively. The higher yield from FLUKA may stem from secondary pion production from the decay of heavier mesons, such as $\eta$, produced at intermediate energy in the interaction. Whereas the average multiplicity primarily follows the number of nucleon-nucleon interactions in the collision, nonlinear effects occur near the $\pi^{0}$ production threshold. They are visible in Figure 1, in particular for the p-He interaction.

For the bremsstrahlung cross section, we have followed the formulation of [11] for electron kinetic energies above $2 \mathrm{MeV}$, interpolating the $\phi_{1}$ and $\phi_{2}$ functions for hydrogen and helium atoms from [12, table II, Hartree-Fock solution for He] onto our grid of electron and $\gamma$-ray energies. We have used the Schiff formula for heavier atoms and set the minimum Fano-Sauter value to the cross section when the emerging $\gamma$-ray energy approaches the incident electron kinetic energy.

We have restricted the choice of target gas nuclei to hydrogen and helium for hadronic production and to $\mathrm{H}, \mathrm{He}, \mathrm{C}, \mathrm{N}, \mathrm{O}, \mathrm{Ne}, \mathrm{Mg}, \mathrm{Si}, \mathrm{S}, \mathrm{Fe}$, and $\mathrm{Ni}$ for bremsstrahlung radiation since those gas components, with local interstellar abundances from [13], dominate the $\gamma$-ray yield. 

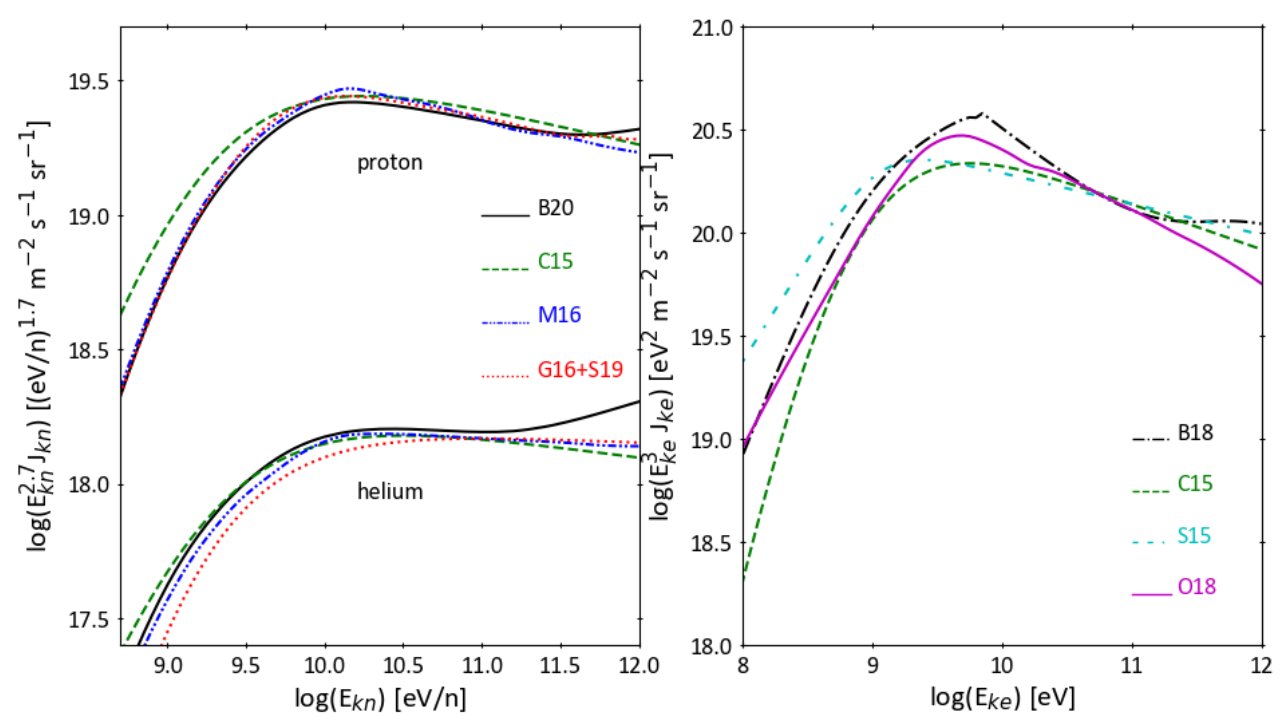

Figure 2: Comparison of the local interstellar spectra (LIS), incident on the heliopause, inferred for CR protons and helium (left, from [5, 10, 15-17]) and for all CR electrons (right, from [15, 19-21].

\section{Cosmic-ray spectra near the Sun}

The heliospheric propagation of CRs leads to a marked flux reduction below $\sim 50 \mathrm{GV}$ in rigidity. The LIS, incident on the heliopause, have been measured aboard Voyager 1 and 2 at kinetic energies $\$ 700 \mathrm{MeV} / \mathrm{n}$ for nuclei and $\leq 74 \mathrm{MeV}$ for electrons [14]. At higher energy, they have been inferred from direct particle data taken inside the Solar System, using either the force-field approximation for solar demodulation with a time-dependent potential [e.g. 15, 16], or solving the Parker transport equation in a time variable configuration of the heliosphere [e.g. 10, 17, 21]. LIS estimates have thus been obtained by purely demodulating the Solar-System data [e.g. 16, 17], by combining solar demodulation and the $\gamma$-ray emissivity of the gas in nearby interstellar clouds [15], or by combining solar demodulation and more distant information on CR propagation in the Galaxy ( $\gamma$-ray and radio maps, secondary to primary spallation ratios, antiprotons, etc) [e.g. 5, 10, 21].

The $\gamma$-ray emissivity of the interstellar gas is best measured in the $0.05-30 \mathrm{GeV}$ range. Figure 2 shows that the proton and helium spectra compare well in the $1-300 \mathrm{GeV} / \mathrm{n}$ band that contributes most of these $\gamma$ rays. The hardening break confirmed by CALET near 200-300 GeV/n [18] was modelled only in the 'B20' curve shown from [10] in this set. At lower energies, the proton spectra differ by less than $12 \%$ if we discard the low-energy part of the Casandjian (2015) spectrum which was not constrained by Voyager data like the others. The difference in helium is more pronounced as it varies from $45 \%$ down to $10 \%$ and up again to $20 \%$ with increasing energy across the band. The comparison in Figure 2 shows that the addition of indirect information on propagated CRs in the Galaxy does not strongly affect the result, the latter being largely driven by Solar-System data and solar demodulation.

The all-electron spectrum is more uncertain, in particular at energies below $1 \mathrm{GeV}$ which are most relevant for Fermi LAT observations, even if we take into account that the two most extreme 


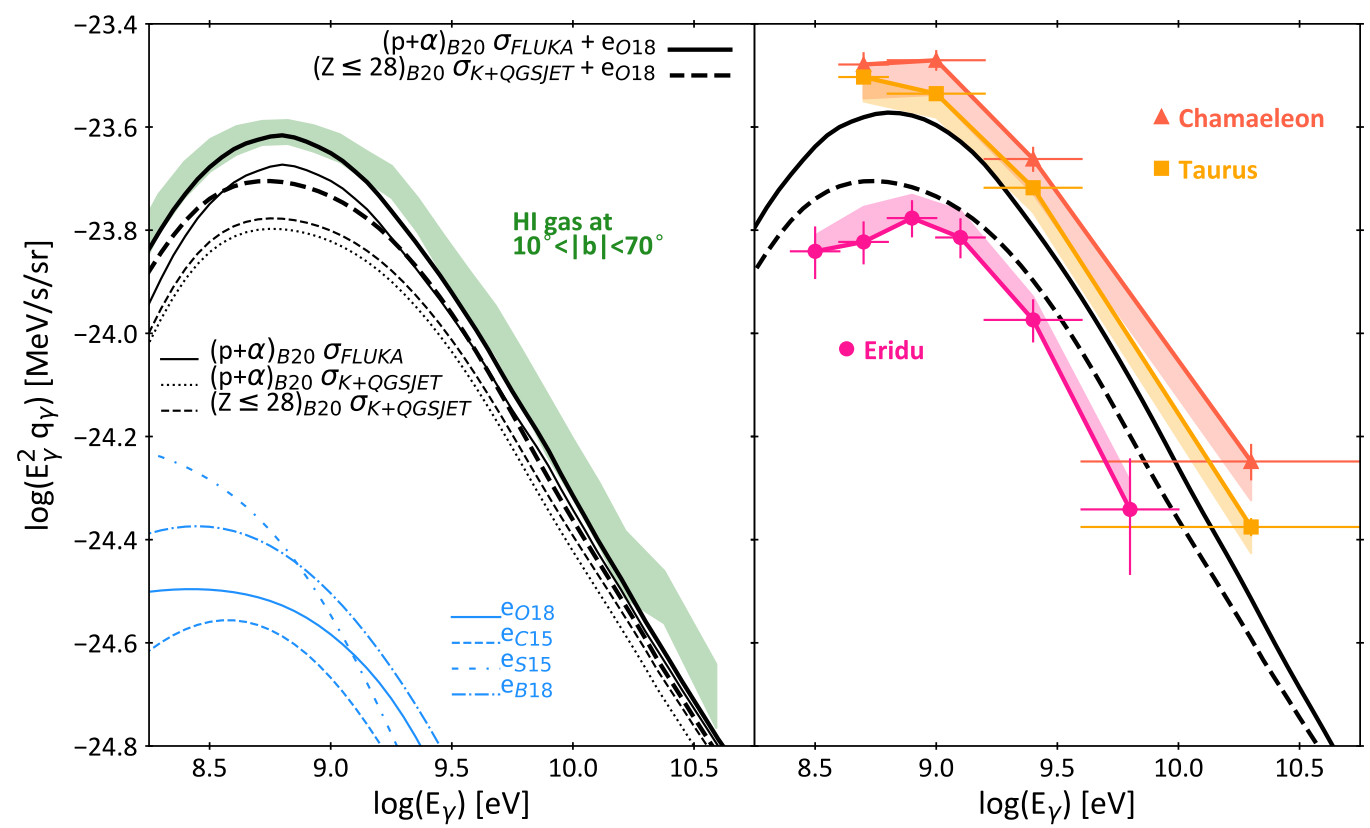

Figure 3: Emissivity spectra per gas nucleon for hadronic (thin black), bremsstrahlung (thin blue), and total (thick) emission, using the FLUKA (solid) and 'K+QGSJET' (dashed) cross sections. The total emissivities are calculated for the CR spectra found near the Solar System (LIS) in nuclei (from [10]) and all electrons (from [19]). See Figure 2 for the other electron references. The emissivities are compared with the average found in the atomic gas within $\sim 1 \mathrm{kpc}$ from the Sun (left plot, shaded green area for the satistical uncertainty, from [15]) and in the nearby clouds of Eridu, Taurus, and Chamaeleon (right plot, with statistical uncertainties as error bars and the impact of systematic uncertainties in the Hi mass as shaded areas, from [4, 22, 23]).

spectra at low energy were not constrained by Voyager data. Indirect information plays an important role to constrain the spectrum at intermediate energies as spectra brighter than the Orlando (2018) one in the $1-10 \mathrm{GeV}$ band are disfavoured by the radio and $\gamma$-ray maps [19].

\section{The cosmic-ray deficit in the Eridu cirrus}

Figure 3 shows the $\gamma$-ray emissivities one would expect in nearby clouds if the CR spectra pervading them were the same as the LIS reaching the heliophere. For the total emissivity, we took the input CR spectra from [10] for nuclei and [19] for all electrons. The choice of the proton and helium spectra from [5] would yield a 10\% larger emissivity. We have used the 'K+QGSJET' cross section to illustrate the $\sim 5 \%$ gain in hadronic emissivity if one includes interactions with CR nuclei heavier than helium (see Figure 3, left). We have also used the FLUKA simulation to check that interactions with interstellar $\mathrm{C}, \mathrm{N}, \mathrm{O}, \mathrm{Ne}, \mathrm{Mg}$, and $\mathrm{Si}$ atoms only contribute $0.2 \%$ to the hadronic emissivity on hydrogen and helium.

The average emissivity measured with the LAT in the atomic gas lying within about $1 \mathrm{kpc}$ around the Sun (at $10^{\circ} \leq|b| \leq 70^{\circ}$ ) assumes a low Hi spin temperature of $140 \mathrm{~K}$ to partially correct for the self absorption of the gas to the Hı line radiation [15]. Figure 3 (left) illustrates that the current uncertainty in the $\gamma$-ray yield per nuclear interaction makes it impossible to conclude 
whether the average CR spectrum measured in a 1-kpc-wide region around the Sun is comparable or $\sim 25 \%$ superior to that passing the Solar System. It also shows that the bremsstrahlung radiation represents at least $15 \%$ of the total emission at energies near $1 \mathrm{GeV}$ in this region.

Figure 3 (right) compares the low $\gamma$-ray emissivity found in the Eridu cirrus with measurements in two specific nearby clouds on the one hand, and with the range of expectations from the LIS near the Solar System on the other hand. The emissivity measurements have been obtained in the atomic phase of the clouds, at comparable linear resolutions of $0.3-1 \mathrm{pc}$, with the same tracers to map all gas phases, including the dark one, and the same method to model the spatial and spectral distributions of $\gamma$ rays observed by the LAT. The emissivity measurement relies on the spatial correlation between the $\gamma$ rays and the gas column densities in the Hi-bright phase. The Eridu cirrus is located 200-300 pc away from the Sun and at a height of 200-250 pc below the Galactic plane [4]. It shares with the Sun a common location in a long, rather hollow valley of the local ISM that is surrounded by denser, star forming, molecular clouds. In order to compare Eridu with other nearby clouds, we have selected two regions along the valley rim and toward the same valley sector, namely the Chamaeleon complex and the main cloud of the Taurus complex. They respectively lie at distances of $\left(183 \pm 3_{\text {stat }} \pm 9_{\text {syst }}\right) \mathrm{pc}$ and $\left(141 \pm 2_{\text {stat }} \pm 7_{\text {syst }}\right)$ pc from the Sun and at heights of $\sim 50 \mathrm{pc}$ and $\sim 40 \mathrm{pc}$ below the Galactic plane [24]. Figure 3 shows the marked difference observed in $\gamma$-ray emissivity between the light atomic cirrus of Eridu and the more massive molecular clouds flanking the valley. Given the statistical and sytematic uncertainties shown in Figure 3, the emissivity difference reflects a significant difference in CR flux between clouds that are only 200 to 300 pc away from each other.

The CR flux in the Eridu cirrus appears to be half lower than in the nearby massive clouds. Gas mass uncertainties cannot account for this large difference. The Eridu emissivity cannot exceed the upper limit of the shaded area which corresponds to optically thin Hi gas, hence to the very minimum amount of Hi gas present in the cloud. Conversely, adding hidden Hi gas to the Chamaeleon and Taurus clouds would reduce their emissivities in an energy-independent way. The shaded areas show the magnitude of the effect when changing the cloud Hi optical thickness from the optimum value to a much thicker case. One could further add opaque $\mathrm{HI}$ clumps and diffuse $\mathrm{CO}$-dark $\mathrm{H}_{2}$ gas, but this dark phase has a different spatial distribution than the Hi-bright one and it is mapped and included in the analyses as a separate gas component. So is the CO-bright molecular phase. The emissivity of the HI-bright gas is therefore not affected by the presence of the denser gas phases, whether dark or bright, as long as their spatial distributions can be resolved and distinguished. This is the case for the Chamaeleon and Taurus clouds [22, 23]. Doubling the HI-bright gas mass of the Chamaeleon and Taurus clouds to reconcile their emissivities with that of Eridu is therefore implausible.

Because of the uncertainty in $\gamma$-ray yield per nuclear interaction, it is unfortunately impossible at this stage to conclude whether the CR flux reaching the Solar System is close to that of Eridu as both lie in a low-density valley where the large-scale magnetic field is more ordered than in the denser and clumpier clouds surrounding the valley, or whether the CR flux near the Sun compares well with that in the massive clouds and the deficit towards Eridu is specific to its magnetic configuration and/or height off the plane. As seen in dust polarisation toward this sector, the magnetic field in the local valley is mostly parallel to the Galactic disc whereas the filamentary cirrus of Eridu stretches along a different magnetic tube that is highly inclined (at $\sim 45^{\circ}$ ) toward the 
South Galactic pole and halo [4]. The alignment between gas filaments and magnetic-field lines is frequent in this type of cirrus [25], but assessing whether or not such a configuration favours a fast CR diffusion along the filament requires detailed parsec-scale models. As argued in [4] and by Bustard \& Zweibel at this conference, the difference between the CR flux in the filamentary Eridu cirrus and the clumpier and denser Chamaeleon and Taurus clouds is unlikely due to hampered CR penetration into the multi-phase gas, especially at the energies above a few $\mathrm{GeV}$ that are sampled by the LAT data. Moreover, the light Eridu cirrus is much less dense than the Chamaeleon and Taurus clouds where we see $\gamma$ rays produced by CR deep into the dense molecular parts, with the same energy spectrum as in the atomic envelopes. This set of clouds therefore offers an excellent test bed to study CR transport in different types of clouds and over short distance scales of 200 or $300 \mathrm{pc}$.

Acknowledgements: we acknowledge the financial support by the Institut Universitaire de France and LabEx UnivEarthS (ANR-10-LABX-0023 and ANR-18-IDEX-0001) for this work.

\section{References}

[1] Grenier I. A., Black J. H., Strong A. W., 2015, ARA\&A, 53, 199

[2] Yang R.-. zhi ., de Oña Wilhelmi E., Aharonian F., 2014, A\&A, 566, A142

[3] Neronov A., Malyshev D., Semikoz D. V., 2017, A\&A, 606, A22

[4] Joubaud T., et al., 2020, A\&A, 635, A96

[5] Mazziotta M. N., Cerutti F., Ferrari A., Gaggero D., Loparco F., Sala P. R., 2016, APh, 81, 21

[6] Kachelrieß M., Moskalenko I. V., Ostapchenko S., 2019, CoPhC, 245, 106846

[7] Kamae T., Karlsson N., Mizuno T., Abe T., Koi T., 2006, ApJ, 647, 692

[8] Kachelrieß M., Ostapchenko S., 2012, PhRvD, 86, 043004

[9] Mori M., 2009, APh, 31, 341

[10] Boschini M. J., et al., 2020, ApJS, 250, 27

[11] Strong A. W., Moskalenko I. V., Reimer O., 2000, ApJ, 537, 763

[12] Blumenthal G. R., Gould R. J., 1970, RvMP, 42, 237

[13] Asplund M., Grevesse N., Sauval A. J., Scott P., 2009, ARA\&A, 47, 481

[14] Stone E. C., Cummings A. C., Heikkila B. C., Lal N., 2019, NatAs, 3, 1013

[15] Casandjian J.-M., 2015, ApJ, 806, 240

[16] Ghelfi A., Barao F., Derome L., Maurin D., 2016, A\&A, 591, A94

[17] Shen Z.-N., Qin G., Zuo P., Wei F., 2019, ApJ, 887, 132 
[18] Adriani, O., Akaike, Y., Asano, K., et al. 2019, PRL, 122, 181102.

[19] Orlando E., 2018, MNRAS, 475, 2724

[20] Strong A., Fermi-LAT Collaboration, 2015, ICRC, 34, 506

[21] Boschini M. J., et al., 2018, ApJ, 854, 94

[22] Planck Collaboration, Fermi Collaboration, et al., 2015, A\&A, 582, A31

[23] Remy Q., Grenier I. A., Marshall D. J., Casandjian J. M., 2017, A\&A, 601, A78

[24] Zucker C., et al., 2019, ApJ, 879, 125

[25] Clark S. E., Hensley B. S., 2019, ApJ, 887, 136 